\title{
Thermo-Diffusive Flow of Chemically Reacting Fluid in a Saturated Porous Medium for Radiative Heat Flux
}

\author{
N. J. Hazarika ${ }^{1 *}$, S. Ahmed ${ }^{2}$ \\ ${ }^{1}$ Department of Mathematics, Tyagbir Hem Baruah College, Jamugurihat, Sonitpur-784189, Assam, \\ India \\ ${ }^{2}$ Department of Mathematics, Rajiv Gandhi Central University, Rono Hills, Itanagar, Arunachal \\ Pradesh-791112, India
}

Received 10 November 2020, accepted in revised form 23 February 2021

\begin{abstract}
A theoretical investigation has been made for a radiating and chemically reacting viscousfluid of a conducting gas over a vertical porous surface immerse in a saturated porous medium for the impact of magnetic drag-force, buoyancy forces and thermal-diffusion (Soret) order under oscillatory suction and heat absorption. The analytical solutions have been derived for the physical variables related to the governing equations under the appropriate boundary conditions. It is found that the velocity profiles increase with the increasing values of Soret number $\left(S_{0}\right)$ and higher Soret number $\left(S_{0}=5\right)$ shows the large temperature difference. It is noticed that increasing radiation parameter $(R)$ elevated the velocity profiles near the plate. In the effects of Soret number $\left(S_{0}\right)$ the velocity profiles are an increasing function for both the cases of $M=0$ and $M=10$. The validity and accuracy of the flow model is presented and found suitable to proceed the work. In this study, the findings would be useful in many practical areas such as diffusion operations, which involved molecular diffusion of species with molar concentration.
\end{abstract}

Keywords: MHD; Thermal-diffusion; Radiative heat flux; Porous medium.

(C) 2021 JSR Publications. ISSN: 2070-0237 (Print); 2070-0245 (Online). All rights reserved. doi: http://dx.doi.org/10.3329/jsr.v13i2.50425 J. Sci. Res. 13 (2), 507-520 (2021)

\section{Introduction}

Convection flows in porous media have gained significant attention in the field of heat transport in porous media, because it has significant importance in engineering applications such as geothermal systems, solid matrix heat exchangers, thermal insulations, oil extraction and store of nuclear waste materials. These can also be applied to underground coal gasification, ground water hydrology, wall cooled catalytic reactors, energy efficient drying processes and natural convection in earth's crust. The impact of constant surface temperature on free convection currents on the oscillatory flow along a porous surface in porous medium has studied by Hiremath and Patil [1]. A comprehensive account of available information in this field is provided in books by Pop and Ingham [2],

\footnotetext{
*Corresponding author: navahazarika12@gmail.com
} 
Ingham and Pop [3], Vafai [4], Vadasz [5] etc. An experimental analysis has made by Mythreye et al. [6] for a convective heat-mass transfer flow of magnetic fluid past a semiinfinite vertical permeable moving plate due to heat absorption and first order chemical reaction.

Recent developments in hypersonic flights, nuclear power plant, gas turbines and various propulsion devices for aircraft, missiles, satellites and space vehicles have focused attention of researchers on thermal radiation as a mode of energy transfer and emphasized the need for inclusion of radiative transfer in these processes. In this context, several authors [7-13] investigated the interaction of radiation with mixed and natural convection flow past a vertical plate with buoyancy forces either analytically or numerically.

However, the thermal-diffusion (Soret) effect had not taken into account in the abovementioned literature of reviews. This assumption is justified when the concentration level is very low. The flux of mass caused due to temperature gradient is known as Soret effect or thermal-diffusion effect. On the other hand, the energy flux caused by concentration gradient is called Dufour effect. Soret and Dufour effects are important phenomena in areas such as hydrology, petrology and geosciences. Charles Soret first did the experimental investigation of the thermal-diffusion effect on mass transfer related problems in 1879. Thereafter this thermal-diffusion is termed as the Soret effect in honour of Charles Soret. In general, the Soret effect is of a smaller order of magnitude than the effect described in Fick's law and very often, it is neglected in mass transfer process. Though this effect is quite small, but the devices can be arranged to produce very steep temperature gradient so that the separation of components in mixtures is affected. Eckert and Drake [14] have emphasized that in the cases concerning isotope separation and in mixtures between gases with very light molecular weight $\left(\mathrm{H}_{2}, \mathrm{He}\right)$ and for medium molecular weight $\left(\mathrm{N}_{2}\right.$, air $)$, Soret effect is found to be of considerable magnitude such that it cannot be ignored. In view of these above-mentioned studies, the many researchers [15-26] have analyzed theoretically the Soret and Dufour effects in porous medium on heat-mass transfer for convective flow and they presented their numerical solutions with various effects of heat source / sink and suction / injection.

Apart from these studies, investigators [27-33] had investigated analytically and numerically for the heat-mass transport in porous media due to the application of magnetic fluid with various configurations. Hazarika et al. [34] has presented the analytical analysis of $\mathrm{Cu}$-water nano-fluid of natural convection hydro-magnetic heat transport in a Darcian porous regime with diffusion-thermo.

The objective of the present paper is to analyze the effects of Soret number and thermal radiation on free convective periodic heat and mass transport of a viscous incompressible chemically reacting fluid over an infinite porous vertical plate subject to a time dependent suction velocity in presence of a uniform transverse magnetic field and heat generation / absorption. 


\section{Mathematical Formulation}

We consider the unsteady two-dimensional flow of a laminar, incompressible, viscous, electrically conducting, heat absorbing and chemically reacting fluid past a semi-infinite vertical permeable moving plate embedded in a uniform porous medium and subjected to a uniform transverse magnetic field in the presence of Soret and thermal radiation effects.



Fig. A. Flow model and coordinate system.

In rectangular Cartesian co-ordinate system, we take $x$-axis along the plate in the direction of flow and $y$-axis normal to it. In the analysis, induced magnetic field is neglected due to lower magnetic Reynolds number. The flow in the medium is entirely due to buoyancy force cause by temperature difference between the porous plate and the fluid. The governing equations for this investigation are based on the balances of mass, linear momentum, energy and concentration species and they are:

$$
\begin{aligned}
& \frac{\partial \bar{v}}{\partial \bar{y}}=0 \\
& \frac{\partial \bar{u}}{\partial \bar{t}}+\bar{v} \frac{\partial \bar{u}}{\partial \bar{y}}=-\frac{1}{\rho} \frac{\partial \bar{p}}{\partial \bar{x}}+v \frac{\partial^{2} \bar{u}}{\partial \bar{y}^{2}}+g \beta_{T}\left(\bar{T}-\bar{T}_{\infty}\right)+g \beta_{C}\left(\bar{C}-\bar{C}_{\infty}\right)-\frac{\sigma B_{0}^{2} \bar{u}}{\rho}-\frac{v}{\bar{K}} \bar{u} \\
& \frac{\partial \bar{T}}{\partial \bar{t}}+\bar{v} \frac{\partial \bar{T}}{\partial \bar{y}}=\alpha \frac{\partial^{2} \bar{T}}{\partial \bar{y}^{2}}-\frac{1}{\rho C_{p}} \frac{\partial \bar{q}_{r}}{\partial \bar{y}}-\frac{Q_{0}}{\rho C_{p}}\left(\bar{T}-\bar{T}_{\infty}\right) \\
& \frac{\partial \bar{C}}{\partial \bar{t}}+\bar{v} \frac{\partial \bar{C}}{\partial \bar{y}}=D_{m} \frac{\partial^{2} \bar{C}}{\partial \bar{y}^{2}}+D_{T} \frac{\partial^{2} \bar{T}}{\partial \bar{y}^{2}}-\bar{K}_{r}\left(\bar{C}-\bar{C}_{\infty}\right)
\end{aligned}
$$

The suitable boundary conditions of the flow problem are 


$$
\left\{\begin{array}{c}
\bar{u}=\bar{u}_{p}, \bar{T}=\bar{T}_{w}+\varepsilon\left(\bar{T}_{w}-\bar{T}_{\infty}\right) e^{\bar{n} \bar{t}}, \bar{C}=\bar{C}_{w}+\varepsilon\left(\bar{C}_{w}-\bar{C}_{\infty}\right) e^{\bar{n} \bar{t}} \text { at } \bar{y}=0 \\
\bar{u} \rightarrow \bar{U}_{\infty}=U_{0}\left(1+\varepsilon e^{\bar{n} \bar{t}}\right), \bar{T} \rightarrow \bar{T}_{\infty}, \bar{C} \rightarrow \bar{C}_{\infty} \quad \text { as } \bar{y} \rightarrow \infty
\end{array}\right\}
$$

Eq. (1) designates that the suction velocity at the plate surface is a function of time and thus it may be expressed as

$$
\bar{v}=-V_{0}\left(1+\varepsilon A e^{\bar{n} \bar{t}}\right)
$$

Outside the boundary layer, Eq. (2) gives

$$
-\frac{1}{\rho} \frac{d \bar{p}}{d \bar{x}}=\frac{d \bar{U}_{\infty}}{d \bar{t}}+\frac{v}{\bar{K}} \bar{U}_{\infty}+\frac{\sigma}{\rho} B_{0}^{2} \bar{U}_{\infty}
$$

The radiative heat-flux due to Rosseland approximation is

$$
\bar{q}_{r}=-\frac{4 \bar{\sigma}}{3 \bar{a}} \frac{\partial \bar{T}^{4}}{\partial \bar{y}}
$$

where $\bar{\sigma}$ and $\bar{a}$ are respectively the Stefan-Boltzmann constant and the mean absorption co-efficient.

The dimensionless variables are

$$
\left\{\begin{array}{c}
u=\frac{\bar{u}}{U_{0}}, v=\frac{\bar{v}}{V_{0}}, y=\frac{V_{0} \bar{y}}{v}, U_{\infty}=\frac{\bar{U}_{\infty}}{U_{0}}, U_{p}=\frac{\bar{u}_{p}}{U_{0}}, \quad t=\frac{\bar{t} V_{0}^{2}}{v}, \\
K_{r}=\frac{\bar{K}_{r} v}{V_{0}^{2}}, \theta=\frac{\bar{T}-\bar{T}_{\infty}}{\bar{T}_{w}-\bar{T}_{\infty}}, \phi=\frac{\bar{C}-\bar{C}_{\infty}}{\bar{C}_{w}-\bar{C}_{\infty}}, \quad n=\frac{\bar{n} v}{V_{0}^{2}}, \quad K=\frac{\bar{K} V_{0}^{2}}{v^{2}}, \\
Q=\frac{v Q_{0}}{\rho C_{p} V_{0}^{2}}, \operatorname{Pr}=\frac{v \rho C_{p}}{k}=\frac{v}{\alpha}, S c=\frac{v}{D_{m}}, M=\frac{\sigma B_{0}^{2} v}{\rho V_{0}^{2}}, \quad R=\frac{4 \bar{\sigma} \bar{T}_{\infty}^{3}}{k \bar{a}}, \\
S_{0}=\frac{D_{T}\left(\bar{T}_{w}-\bar{T}_{\infty}\right)}{v\left(\bar{C}_{w}-\bar{C}_{\infty}\right)}, G r=\frac{g \beta_{T} v\left(\bar{T}_{w}-\bar{T}_{\infty}\right)}{U_{0} V_{0}^{2}}, G m=\frac{g \beta_{C} v\left(\bar{C}_{w}-\bar{C}_{\infty}\right)}{U_{0} V_{0}^{2}},
\end{array}\right\}
$$

In view of Eqs. (6) - (9), Eqs. (2) - (4) reduce to the following dimensionless form:

$$
\begin{aligned}
& \frac{\partial u}{\partial t}-\left(1+\varepsilon A e^{n t}\right) \frac{\partial u}{\partial y}=\frac{d U_{\infty}}{d t}+\frac{\partial^{2} u}{\partial y^{2}}+G r \theta+G m \phi+N\left(U_{\infty}-u\right) \\
& \frac{\partial \theta}{\partial t}-\left(1+\varepsilon A e^{n t}\right) \frac{\partial \theta}{\partial y}=\frac{1}{\operatorname{Pr}}\left[1+\frac{4 R}{3}\right] \frac{\partial^{2} \theta}{\partial y^{2}}-Q \theta \\
& \frac{\partial \phi}{\partial t}-\left(1+\varepsilon A e^{n t}\right) \frac{\partial \phi}{\partial y}=\frac{1}{S c} \frac{\partial^{2} \phi}{\partial y^{2}}+S_{0} \frac{\partial^{2} \theta}{\partial y^{2}}-K_{r} \phi
\end{aligned}
$$

where

$$
N=M+\frac{1}{K}
$$

The dimensionless form of the boundary condition becomes 


$$
\left\{\begin{array}{c}
u=U_{p}, \theta=1+\varepsilon e^{n t}, \phi=1+\varepsilon e^{n t} \text { at } y=0 \\
u \rightarrow U_{\infty}=1+\varepsilon e^{n t}, \theta \rightarrow 0, \phi \rightarrow 0 \text { as } y \rightarrow \infty
\end{array}\right\}
$$

\section{Solution of the Problem}

The analytical solutions of the Eqs. (10) - (12) due to classical perturbation technique may represents as

$$
f(y, t)=f_{0}(y)+\varepsilon e^{n t} f_{1}(y, t)+0\left(\varepsilon^{2}\right)+\cdots \cdots \cdots \cdots \cdots \cdots \cdots \cdots \cdots \cdots \cdots
$$

where $f$ stands for $u, \theta$ or $\phi$.

On using (14), the Eqs. (10) - (12) lead to

$$
\begin{aligned}
& \left\{\begin{array}{c}
u_{i}^{\prime \prime}+u_{i}^{\prime}-(N+j) u_{i}=-l u_{0}^{\prime}-\operatorname{Gr} \theta_{i}-G m \phi_{i}-(N+j) \\
\text { where } i=0: j=l=0 ; \text { where } i=1: j=n, l=A
\end{array}\right\} \\
& \left\{\begin{array}{c}
(3+4 R) \theta_{i}^{\prime \prime}+3 \operatorname{Pr} \theta_{i}^{\prime}-3 \operatorname{Pr}(j+Q) \theta_{i}=-3 \operatorname{lPr} \theta_{i}^{\prime} \\
\text { where } i=0: j=l=0 ; \text { where } i=1: j=n, l=A
\end{array}\right\} \\
& \left\{\begin{array}{c}
\phi_{i}^{\prime \prime}+S c \phi_{i}^{\prime}-S c\left(j+K_{r}\right) \phi_{i}=-l S c \phi_{0}^{\prime}-S_{0} S c \theta_{i}^{\prime \prime} \\
\text { where } i=0: j=l=0 ; \text { where } i=1: j=n, l=A
\end{array}\right\}
\end{aligned}
$$

The reduced boundary conditions are

$$
\left\{\begin{array}{c}
u_{0}=U_{p}, u_{1}=0, \theta_{0}=1, \theta_{1}=1, \phi_{0}=1, \phi_{1}=1 \text { at } \mathrm{y}=0 \\
u_{0}=1, u_{1}=1, \theta_{0} \rightarrow 0, \theta_{1} \rightarrow 0, \phi_{0} \rightarrow 0, \phi_{1} \rightarrow 0 \text { as } y \rightarrow \infty
\end{array}\right\}
$$

The solutions of Eqs. (15) - (17) under the boundary conditions (18) are

$$
\begin{aligned}
& u_{0}(y)=1+A_{9} e^{-m_{5} y}-A_{7} e^{-m_{1} y}-A_{8} e^{-m_{3} y}, \\
& u_{1}(y)=\left\{\begin{array}{c}
1+A_{15} e^{-m_{6} y}+A_{10} e^{-m_{5} y}-A_{11} e^{-m_{1} y} \\
+A_{13} e^{-m_{2} y}-A_{14} e^{-m_{4} y}
\end{array}\right\}, \\
& \theta_{0}(y)=e^{-m_{1} y}, \theta_{1}(y)=e^{-m_{2} y}+A_{1}\left(e^{-m_{1} y}-e^{-m_{2} y}\right), \\
& \phi_{0}(y)=e^{-m_{3} y}+A_{2}\left(e^{-m_{3} y}-e^{-m_{1} y}\right), \\
& \phi_{1}(y)=A_{6} e^{-m_{4} y}+A_{3} e^{-m_{3} y}-A_{4} e^{-m_{1} y}-A_{5} e^{-m_{2} y} .
\end{aligned}
$$

\subsection{Skin-friction}

Knowing the velocity field [Eqs. (19), (20)], the Skin-friction at the plate can be obtained in non-dimensional form is

$$
\begin{aligned}
& C_{f}=\left[\frac{\tau_{w}}{\rho U_{0} V_{0}}\right]=\left(\frac{\partial u}{\partial y}\right)_{y=0} \\
& =\left[-m_{5} A_{9}+m_{1} A_{7}+m_{3} A_{8}+\varepsilon e^{n t}\left(\begin{array}{l}
-m_{6} A_{15}-m_{5} A_{10}+m_{1} A_{11} \\
+m_{3} A_{12}-m_{2} A_{13}+m_{4} A_{14}
\end{array}\right)\right]
\end{aligned}
$$




\subsection{Nusselt number}

From temperature field Eq. (21), the rate of heat transfer co-efficient can be obtained in the non-dimensional form in terms of Nusselt number is

$$
N u R e_{x}^{-1}=-\left(1+\frac{4 R}{3}\right)\left(\frac{\partial \theta}{\partial y}\right)_{y=0}=\left(1+\frac{4 R}{3}\right)\left[m_{1}+\varepsilon e^{n t}\left\{m_{1} A_{1}+m_{2}\left(1-A_{1}\right)\right\}\right]
$$

\subsection{Sherwood number}

From concentration field [Eqs. (22), (23)], the rate of mass transfer co-efficient can be obtained in the non-dimensional form in terms of Sherwood number is

$$
\begin{aligned}
& \operatorname{ShRe}_{x}^{-1}=-\left(\frac{\partial \phi}{\partial y}\right)_{y=0} \\
& \quad=\left[m_{3}\left(1+A_{2}\right)-m_{1} A_{2}+\varepsilon e^{n t}\left(m_{4} A_{6}+m_{3} A_{3}-m_{1} A_{4}-m_{2} A_{5}\right)\right],
\end{aligned}
$$

where $R e_{x}=\frac{V_{0} x}{v}$ is the local Reynolds number.

\section{Accuracy}

In order to check the accuracy of the employed Perturbation method, comparisons with the previously published work of Mythreye et al. [6] have been done. In this present flow model when Soret effect $\left(S_{0}=0\right)$ and thermal-radiation parameter $(R=0)$, the work reduced to the flow model done by Mythreye et al. [6].

Table 1. Comparison of the present results with the results done by Mythreye et al. [6] with the effects of heat absorption $(Q)$ on flow velocity $(u)$ when $\operatorname{Pr}=0.71, S c=0.6, G r=2$, $G m=1, M=0, K=0.5, U_{P}=0.5, A=0.5, t=0.5, n=0.1, \varepsilon=0.2$.

\begin{tabular}{cllllll}
\hline \multirow{2}{*}{$y$} & \multicolumn{3}{c}{ Present work } & \multicolumn{3}{c}{ Mythreye et al. [6] } \\
\cline { 2 - 7 } & $Q=0$ & $Q=2$ & $Q=4$ & $Q=0$ & $Q=2$ & $Q=4$ \\
\hline 0 & 0.5 & 0.5 & 0.5 & 0.5 & 0.5 & 0.5 \\
2 & 1.80585 & 1.42948 & 1.36691 & 1.7996 & 1.41769 & 1.35472 \\
4 & 1.50549 & 1.24897 & 1.23248 & 1.50134 & 1.24524 & 1.22942 \\
6 & 1.3587 & 1.21623 & 1.2131 & 1.35644 & 1.21539 & 1.21256 \\
8 & 1.28668 & 1.21116 & 1.21036 & 1.2855 & 1.211 & 1.21055 \\
10 & 1.25003 & 1.21039 & 1.21031 & 1.24942 & 1.21036 & 1.21029 \\
\hline
\end{tabular}

Table 2. Comparisons of the present results with the results done by Mythreye et al. [6] with the effects of various thermal Grashof number $(G r)$ on skin-friction $(\tau)$ when $\operatorname{Pr}=0.71, S c=0.6$, $G m=1, K=0.5, U_{P}=0.5, A=0.5, t=0.5, n=0.1, \varepsilon=0.2$.

\begin{tabular}{lllllll}
\hline \multirow{2}{*}{$M$} & \multicolumn{3}{c}{ Present work } & \multicolumn{3}{c}{ Mythreye et al. [6] } \\
\cline { 2 - 7 } & $G r=0$ & $G r=2$ & $G r=4$ & $G r=0$ & $G r=2$ & $G r=4$ \\
\hline 0 & 2.01812 & 4.66395 & 7.30978 & 1.94506 & 4.59089 & 7.23672 \\
2 & 2.11437 & 3.46314 & 4.81192 & 2.09339 & 3.44216 & 4.79093 \\
4 & 2.35857 & 3.39417 & 4.42977 & 2.34587 & 3.38147 & 4.41707 \\
\hline
\end{tabular}




\begin{tabular}{cllllll}
\hline 6 & 2.58818 & 3.4628 & 4.33741 & 2.579 & 3.45361 & 4.32823 \\
8 & 2.79935 & 3.57138 & 4.34342 & 2.79213 & 3.56416 & 4.3362 \\
10 & 2.99475 & 3.69397 & 4.3932 & 2.98879 & 3.68801 & 4.38724 \\
\hline
\end{tabular}

\section{Results and Discussion}

In this work, we have investigated the two-dimensional flow model of free convective unsteady flow with periodic heat and mass transfer over a flat porous plate in a Darcian porous regime under oscillatory suction and free stream velocities in presence of Soret and radiation effects. Throughout the discussion all the numerical calculations have been done for the real constants $\operatorname{Pr}=0.71, S c=0.6, G r=10, G m=5, M=1, K=5$, $Q=1, K_{r}=0.5, S_{0}=0.2, U_{P}=0.5, A=0.5, t=1, n=0.2, \varepsilon=0.002$.

The effects of radiation parameter $(R)$ on the flow velocity $(u)$ is presented in Fig. 1. An increase in $R$ from 0 through 1, 2 to 4 , the velocity profiles are elevated near the plate and diminishing in the free stream velocity. Significantly, the greater value of $u$ has attained for $R=4$ rather than $R=0$.

Fig. 2 is a plot of velocity profiles for different values of Soret number $\left(S_{0}\right)$. The Soret number signifies the ratio of temperature difference to the concentration. Hence, the bigger Soret number $\left(S_{0}=5\right)$ stands for a large temperature difference and precipitous gradient. In this Fig. it is clear that the velocity profiles increase with the increasing the values of Soret number $\left(S_{0}\right)$. Thus, the fluid velocity rises due to greater thermal diffusion factor.

The effects of heat source/generation $(Q)$ on the velocity distribution are shown in Fig. 3. The term $Q_{0}\left(\bar{T}-\bar{T}_{\infty}\right)$ is assumed to be the amount of heat generated or absorbed per unit volume. $Q_{0}$ is a constant, which may take on either positive or negative values when $\bar{T}>\bar{T}_{\infty}$, the source term $Q_{0}>0$ and heat sink when $Q_{0}<0$. From this Fig., it is seen that the heat is absorbed due to the buoyancy forces, which induces the flow rate to decrease giving reduction to the decrease in the velocity profiles.

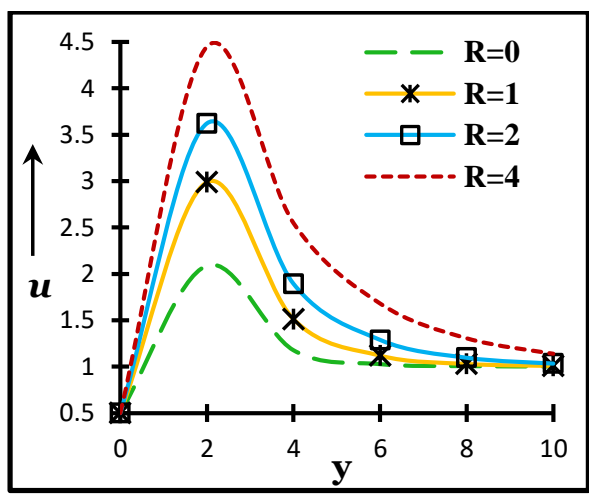

Fig. 1. Velocity profiles for $R$.

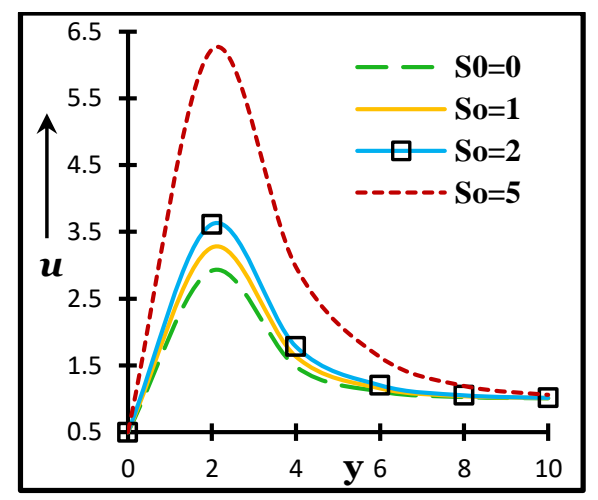

Fig. 2. Velocity profiles for $S_{0}$. 
The variation of velocity distribution of the flow field with the generative chemical reaction $\left(K_{r}\right)$ is shown in Fig. 4. It is observed from this Fig. that the velocity distribution decreases at all points of the flow field with increasing in the chemical reaction parameter. This shows that the generative reaction with higher values of $K_{r}$ have the retarding effect on the velocity distribution of the flow field.

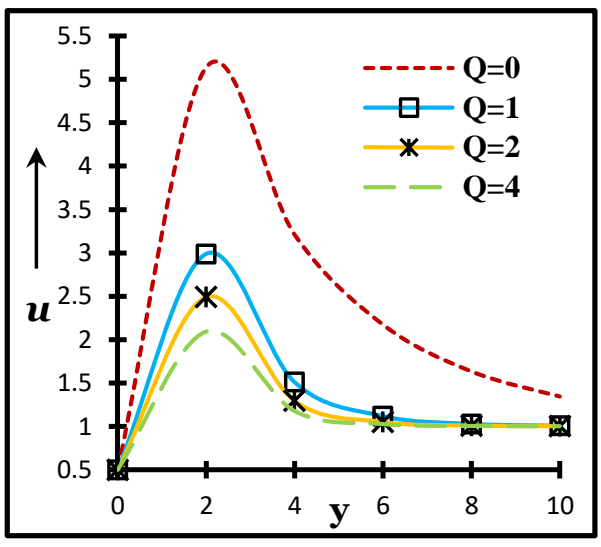

Fig. 3. Velocity profiles for $Q$.

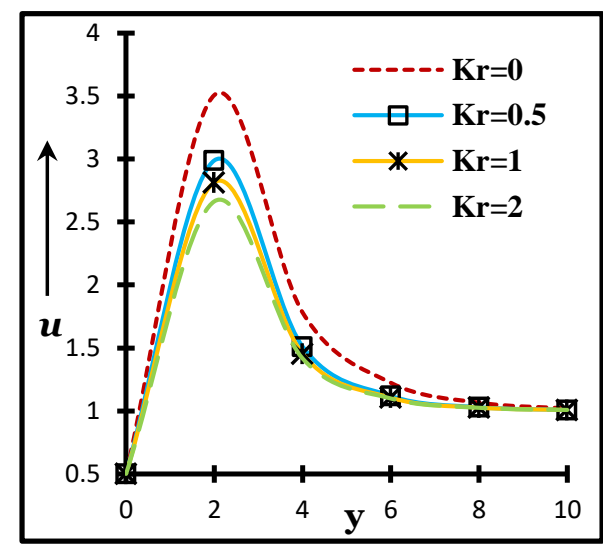

Fig. 4. Velocity profiles for $K_{r}$.

Fig. 5 show the variation of dimensionless temperature profiles with y for different values of radiation parameter $(R)$. It shows that the temperature increases with increasing radiation parameter. This is due to the fact that the increase in the values of thermal radiation parameter increases the flux of energy transport to the fluid and accordingly increases the fluid temperature in the thermal boundary layer. Thus, it is found that the effect of thermal radiation is to enhance heat transfer and due to which there is an increase in the thermal boundary layer thickness.

Fig. 6 depicts the variation of dimensionless temperature profiles for different values of heat generation / absorption rate $(Q)$. The positive sign indicates the heat absorption whereas negative means heat generation. In this Fig. we may realize that the temperature profiles decrease with increasing the parameter $(Q)$, which results in decreasing the thermal boundary layer thickness with stronger $Q$.

The variation of Concentration distribution of the flow field with the diffusion of the foreign mass $\left(S_{0}\right)$ is presented in Fig. 7 in absence of $K_{r}, R$ and $Q$. It is observed that the concentration distribution strongly elevated at all points of the flow field with increasing in the Soret number $\left(S_{0}\right)$. This shows that the diffusive spices with higher value of Soret number have an enhancing effect on the concentration distribution of the flow field. It is interesting to note that the higher values of Soret number $\left(S_{0}>1\right)$ have the tendency in formation of peak concentration profiles near the plate. 


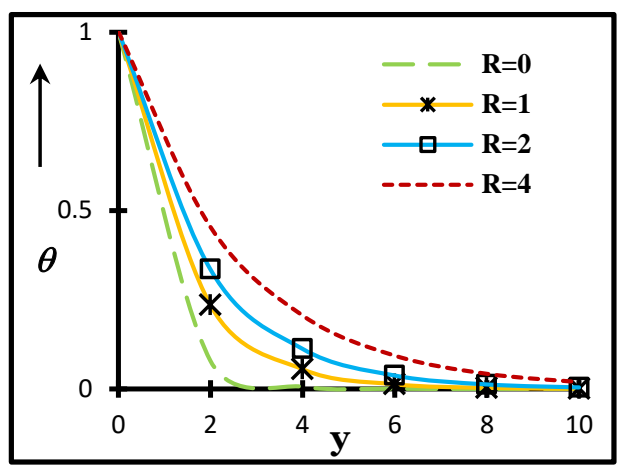

Fig. 5. Temperature profiles for $R$.

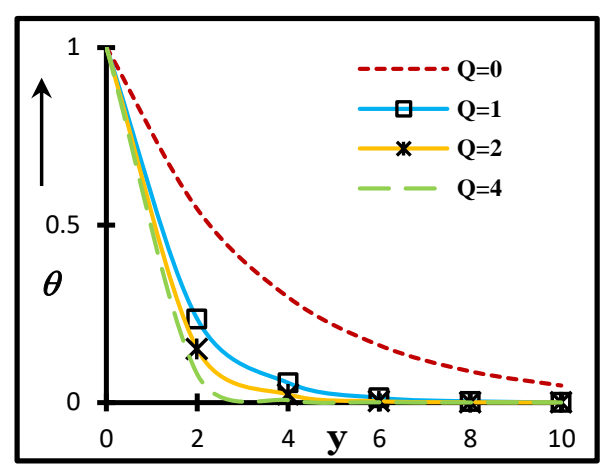

Fig. 6. Temperature profiles for $Q$.

The variation of velocity gradient distribution at the plate $y=0$ for different values of $S_{o}$ and $M$ are shown in Fig. 8. It is seen that there is substantial increase in $(\tau)$ at the plate $\mathrm{y}=0$ for the increasing effects of $S_{0}$, whereas no significant effects have been observed in $\tau$ when $M>2$. However, the skin-friction enhanced for the region $0 \leq M \leq 2$, but for $M>2$ the skin-friction has no effects in the boundary layer.

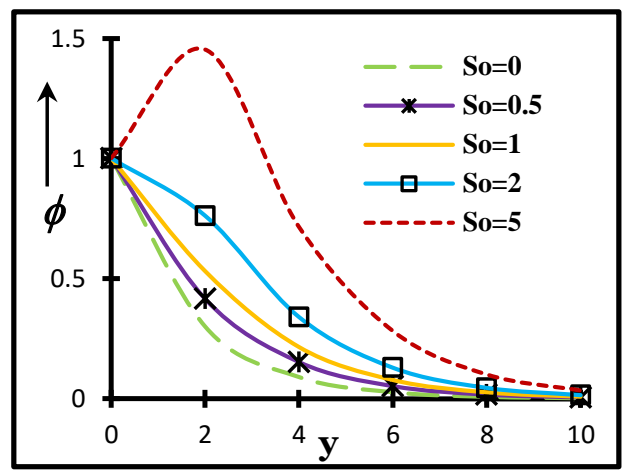

Fig. 7. Concentration profiles for $S_{0}$.

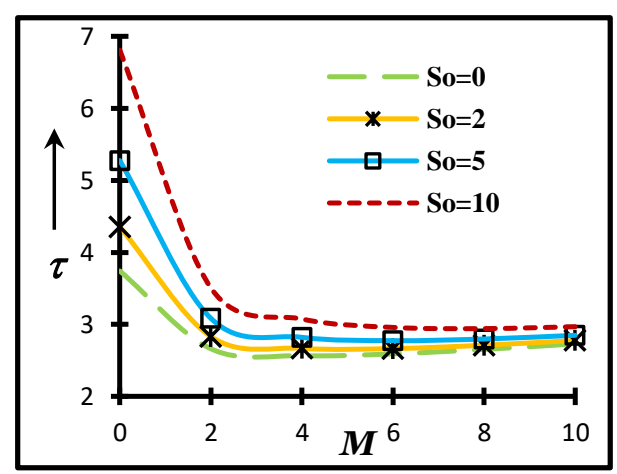

Fig. 8 Skin-friction for $S_{0}$ and $M$.

Fig. 9 depicts the rate of heat transfer responses for various radiation parameter $(R)$ and heat absorption parameter $(Q)$. When $Q$ increases, it is observed that the rate of heat transfer gradually increases. However, there is a substantial increase in the rate of heat transfer due to the effects of thermal radiation $(R)$. For higher radiation $(R=5)$ the thermal energy is boosted rather than the no thermal radiation $(R=0)$.

In Fig. 10 the evolution of Sherwood number distribution $(S h)$ with the effects of Soret number $\left(S_{0}\right)$ and thermal radiation $(R)$ is shown. With an increase in $\left(S_{0}\right)$ the magnitude of mass transfer gradient at the plate is reduces due to the temperature difference to the concentration. However, there is a progressive increasing in mass transfer gradient indicating that thermal radiation exerts a stronger effect than the Soret 
effect. Significantly, the negative values of Sherwood number $(S h)$ have been observed at $S_{0}=3$ and $S_{0}=5$ for $R<2$.

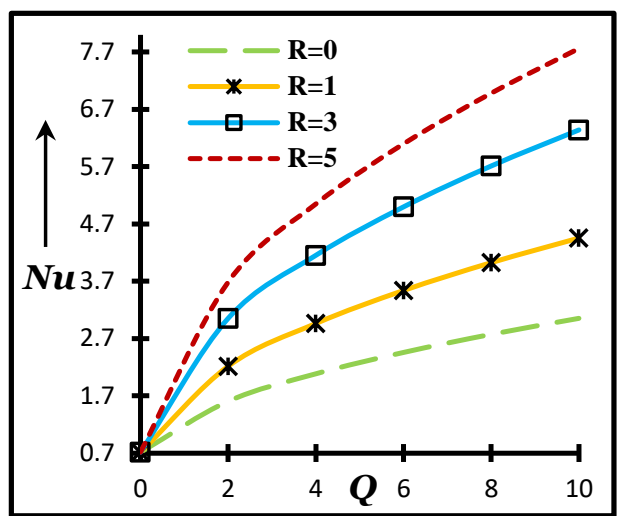

Fig. 9. Nusselt number for $R$ and $Q$.

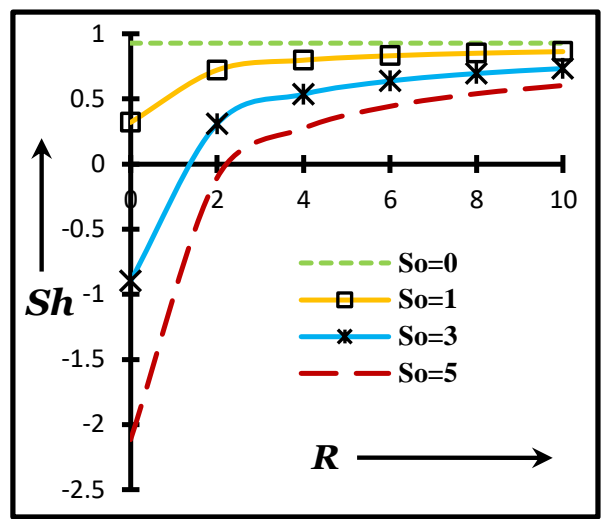

Fig. 10. Sherwood number for $S_{0}$ and $R$.

The influence of magnetic body force $(M)$ and Soret number $\left(S_{0}\right)$ on the velocity distributions with distant normal to the plate (transverse co-ordinate y) is presented in Fig. 11. The hydromagnetic term in the dimensionless Eq. (11), $-M u$ is a linear drag force term. With increasing magnetic field strength $B_{0}, M$ is increased and this serves to decelerate the flow along the plate. The strong inhabiting effect of magnetic field is therefore evident. Moreover, the velocity profiles are elevated due to the effects of Soret number for both the cases of $M=0$ and $M=10$. Significantly velocity overshoot near the plate have been observed for the pure hydrodynamic fluid $(M=0)$.

The variations of radiation and heat generation / absorption on the temperature distributions against the transverse distance y are plotted in the Fig. 12 . When $Q=0.5$ i.e. for heat generation, the temperature distribution are enhanced, therefore the thermal boundary layer thickness gradually increased. On the other hand, the temperature distributions are diminished from heat absorption to heat generation near the plate $(0 \leq y \leq 4)$ but away the plate $y>4$ the reverse effect has been observed.

The effects of Soret number $\left(S_{0}\right)$ and chemical reaction $\left(K_{r}\right)$ on the concentration distribution are presented in Fig. 13. Due to generative chemical reaction $\left(K_{r}=0.5\right)$, the concentration distribution is enhanced by the increasing effect of Soret number $\left(S_{0}\right)$. Moreover, an increasing Soret number the concentration distribution has negative increasing values for destructive chemical reaction when $\mathrm{y}>2$. Interestingly, for destructive chemical reaction $\left(K_{r}=-0.5\right)$, the Soret number enhances the concentration distribution near the plate. However, the concentration distribution changes from destructive to generative chemical reaction regions. 


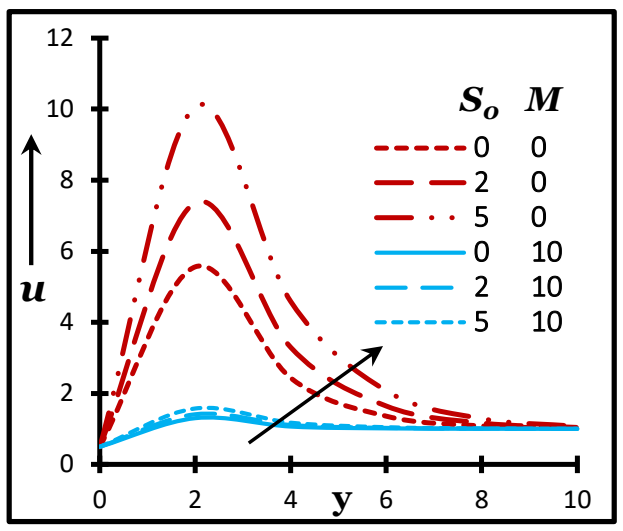

Fig. 11. Velocity profiles for $S_{0}$ and $M$.

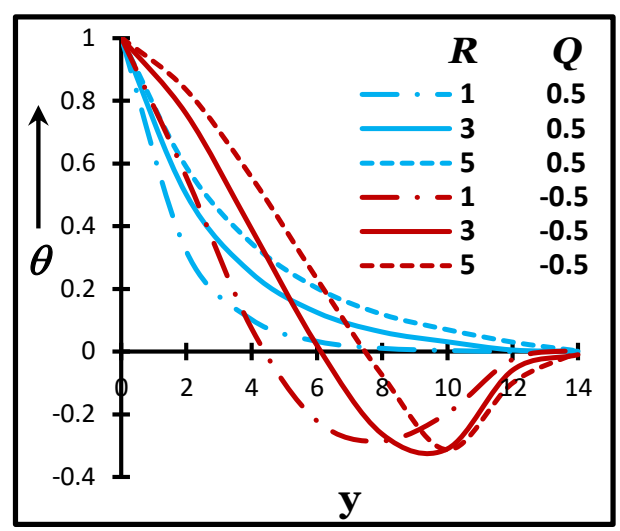

Fig. 12. Temperature profiles for $R$ and $Q$.

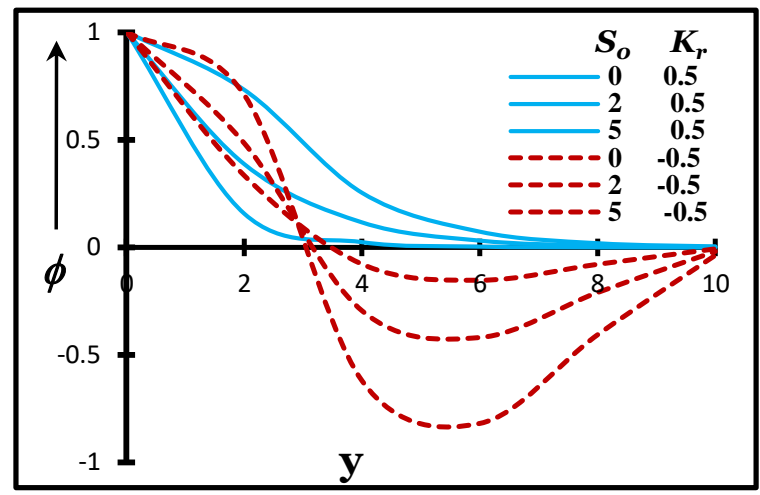

Fig. 13. Concentration profiles for $S_{0}$ and $K_{r}$.

\section{Conclusion}

In this paper, the problem of unsteady boundary layer, heat and mass transfer flow of a viscous incompressible electrically-conducting fluid through porous medium over an infinite vertical plate immersed in a Darcian porous plate with thermal radiation and Soret effects has been considered in presence of heat source / sink and first order chemical reaction. The resulting partial differential equations were transformed into a set of ordinary differential equations using two-term series and solved in closed form. Analytical solutions of the closed form results were performed and some graphical results were obtained to illustrate the details of the flow and heat and mass transfer characteristics and their dependence on some of the physical parameters. The main results of the present study can be concluded as follows:

- the effect of increasing values of radiation is to accelerate the flow velocity as well as same behavior has been observed in the thermal boundary layer and in the rate of heat transfer. 


\section{Thermo-Diffusive Flow of Chemically Reacting Fluid}

- the velocity profiles and concentration boundary layer have been increased in the increasing effects of Soret number and opposite trends have been shown in the skinfriction and Sherwood number.

- due to heat absorption the flow velocity as well as the temperature profiles has been decelerated.

- with increasing values of chemical reaction, the flow velocity enhanced.

- the flow velocity is accelerated with the increasing values of Soret number for both the cases of $M=0$ and $M=10$.

- due to generative chemical reaction the concentration boundary layer enhanced by the increasing effects of Soret number. For destructive chemical reaction, the Soret number enhances the concentration profiles near the plate.

\section{Nomenclature}

\begin{tabular}{|c|c|c|c|}
\hline $\bar{x}$ & dimensional distances along the plate & $\bar{y}$ & $\begin{array}{l}\text { dimensional distances perpendicular to } \\
\text { the plate }\end{array}$ \\
\hline $\bar{u}$ & components of velocity in $\bar{x}$ direction & $\bar{v}$ & components of velocity in $\bar{y}$ direction \\
\hline $\bar{t}$ & dimensional time & $\bar{p}$ & pressure \\
\hline$g$ & gravitational acceleration & $\bar{T}$ & $\begin{array}{l}\text { dimensional temperature of the fluid in } \\
\text { the boundary layer }\end{array}$ \\
\hline $\bar{C}$ & $\begin{array}{l}\text { dimensional concentration in the } \\
\text { boundary layer }\end{array}$ & $\bar{T}_{w}$ & temperature of the fluid at the wall \\
\hline $\bar{C}_{w}$ & molar species concentration at the wall & $\bar{T}_{\infty}$ & temperature at the free stream \\
\hline $\bar{C}_{\infty}$ & $\begin{array}{l}\text { molar species concentration at the free } \\
\text { stream }\end{array}$ & $B_{0}$ & the magnetic induction \\
\hline $\bar{K}$ & permeability of porous medium & $C_{p}$ & specific heat at constant pressure \\
\hline $\bar{q}_{r}$ & the radiative heat flux & $Q_{0}$ & heat absorption coefficient \\
\hline$D_{m}$ & molecular diffusivity & $D_{T}$ & coefficient of thermal diffusivity \\
\hline $\bar{K}_{r}$ & the chemical reaction & $\bar{u}_{p}$ & dimensional plate velocity \\
\hline $\bar{U}_{\infty}$ & dimensional free stream velocity & $U_{0}$ & scale of free stream velocity \\
\hline$V_{0}$ & scale of suction velocity & $U_{p}$ & dimensionless plate velocity \\
\hline$U_{\infty}$ & dimensionaless free stream velocity & $A$ & suction parameter \\
\hline$n$ & dimensionless exponential index & $G r$ & thermal Grashof number \\
\hline$G m$ & solutal Grashof number & $\operatorname{Pr}$ & Prandtl number \\
\hline$S c$ & Schmidt number & $M$ & magnetic field parameter \\
\hline$R$ & thermal radiation & $Q$ & heat source parameter \\
\hline$K$ & permeability parameter & $N$ & dimensionless field parameter \\
\hline$K_{r}$ & chemical reaction parameter & $S_{0}$ & Soret number \\
\hline$C_{f}$ & Skin-friction & $\mathrm{Nu}$ & Nusselt number \\
\hline$S h$ & Sherwood number & & \\
\hline
\end{tabular}

\section{Greek alphabet}

\begin{tabular}{clcl}
\hline$\alpha$ & fluid thermal diffusivity & $\beta_{T}$ & thermal expansion coefficient \\
$\beta_{C}$ & concentration expansion coefficient & $\varepsilon$ & small reference parameter \\
\hline
\end{tabular}




\begin{tabular}{llll}
\hline$\theta$ & dimensionless fluid temperature & $\phi$ & dimensionless fluid concentration \\
$\sigma$ & electrical conductivity & $\rho$ & fluid density \\
$\nu$ & kinemetic viscosity & $\kappa$ & thermal conductivity \\
\hline
\end{tabular}

\section{Subscripts}

$\begin{array}{ll}\mathrm{p} & \text { plate condition } \\ \mathrm{w} & \text { the wall condition } \\ \infty & \text { free stream }\end{array}$

\section{Appendix}

$$
\begin{aligned}
& m_{1}=\frac{1}{2(3+4 R)}\left[3 \operatorname{Pr}+\sqrt{9 P^{2}+12 \operatorname{PrQ}(3+4 R)}\right], m_{2}=\frac{1}{2(3+4 R)}\left[3 \operatorname{Pr}+\sqrt{9 P^{2}+12 \operatorname{Pr}(3+4 R)(n+Q)}\right] \\
& m_{3}=\frac{1}{2}\left[S c+\sqrt{S c^{2}+4 S c K_{r}}\right], m_{4}=\frac{1}{2}\left[S c+\sqrt{S c^{2}+4 S c\left(n+K_{r}\right)}\right], m_{5}=\frac{1}{2}[1+\sqrt{1+4 N}], \\
& m_{6}=\frac{1}{2}[1+\sqrt{1+4(N+n)}], A_{1}=\frac{3 m_{1} \operatorname{Pr} A}{(3+4 R) m_{1}^{2}-3 m_{1} \operatorname{Pr}-3 \operatorname{Pr}(n+Q)}, A_{2}=\frac{m_{1}^{2} S_{0} S c}{m_{1}^{2}-m_{1} S c-S c K_{r}}, \\
& A_{3}=\frac{m_{3} A S c\left(1+A_{2}\right)}{m_{3}^{2}-m_{3} S c-S c\left(n+K_{r}\right)}, A_{4}=\frac{m_{1} S c\left(A A_{2}+m_{1} A_{1} S_{0}\right)}{m_{1}^{2}-m_{1} S c-S c\left(n+K_{r}\right)}, A_{5}=\frac{m_{2}^{2} S_{0} S c\left(1-A_{1}\right)}{m_{2}^{2}-m_{2} S c-S c\left(n+K_{r}\right)}, \\
& A_{6}=1-A_{3}+A_{4}+A_{5}, A_{7}=\frac{G r-A_{2} G m}{m_{1}^{2}-m_{1}-N}, A_{8}=\frac{G m\left(1+A_{2}\right)}{m_{3}^{2}-m_{3}-N}, A_{9}=U_{p}-1+A_{7}+A_{8}, \\
& A_{10}=\frac{m_{5} A_{9} A}{m_{5}^{2}-m_{5}-(N+n)}, A_{11}=\frac{m_{1} A_{7} A+G r A_{1}-G m A_{4}}{m_{1}^{2}-m_{1}-(N+n)}, A_{12}=\frac{m_{3} A_{8} A+G m A_{3}}{m_{3}^{2}-m_{3}-(N+n)}, \\
& A_{13}=\frac{G m A_{5}-G r\left(1-A_{1}\right)}{m_{2}^{2}-m_{2}-(N+n)}, A_{14}=\frac{G m A_{6}}{m_{4}^{2}-m_{4}-(N+n)}, A_{15}=-1-A_{10}+A_{11}+A_{12}-A_{13}+A_{14} .
\end{aligned}
$$

\section{References}

1. P. S. Hiremath and P. M. Patil, Acta Mech., 98, 143 (1993). https://doi:10.1007/BF01174299

2. I. Pop and D. B. Ingham, Convective Heat Transfer: Mathematical and Computational Modelling of Viscous Fluids and Porous Media (Oxford, UK, Pergamon, 2001).

3. Transport Phenomena in Porous Media, ed. D. B. Ingham et al. (Elsevier, Oxford, 2005).

4. K. Vafai, Handbook of Porous Media, $2^{\text {nd }}$ Edition (Taylor and Francis, New York, USA, 2005). https://doi.org/10.1201/9780415876384

5. P. Vadasz, Emerging Topics in Heat and Mass Transfer in Porous Media (Springer, New York, 2008). https://doi.org/10.1007/978-1-4020-8178-1 12

6. A. Mythreye, J. P. Pramod, and K. S. Balamurugan, Procedia Eng. 127, 613 (2015). https://doi.org/10.1016/j.proeng.2015.11.352

7. M. A. Hossain and H. S. Takhar, Heat Mass Transfer 31, 243 (1996). https://doi.org/10.1007/BF02328616

8. A. Raptis and C. Perdikis, Int. J. Non-linear Mech., 41, 527 (2006). https://doi.org/10.1016/j.ijnonlinmec.2005.12.003

9. D. Pal, Meccanica 44, 145 (2009). https://doi.org/10.1007/s11012-008-9155-1

10. M. A. A. EL-Naby, E. M. E. El-barbar, and N. Y. Abdelazem, Appl. Math. Comput. 151, 327 (2004). https://doi.org/10.1016/S0096-3003(03)00344-8 
11. I. J. Uwanta, European J. Sci. Res. 87, 190 (2012).

12. G. Vidyasagar, B. Ramana, and P. B. A. Reddy, J. Global Res. Math. Archives 1, 49 (2013).

13. B. K. Sharma, R. C. Chaudhary, and M. Agarwal, Bull. Calcutta Mathemat. Soc. 100, 529 (2008).

14. E. R. G. Eckert and R. M. Drake, Analysis of Heat and Mass Transfer (McGraw-Hill, NY, 1972).

15. D. T. J. Hurle and E. Jakeman, J. Fluid Mech. 47, 667 (1974).

16. J. K. Platten and G. Chavepeyer, J. Fluid Mech. 60, 305 (1973). https://doi.org/10.1017/S0022112073000182

17. R. Delahaye, A. Bahloul, and P. Vasseur, Int. Commun. Heat Mass Transfer 29, 433 (2002). https://doi.org/10.1016/S0735-1933(02)00340-8

18. M. Ouriemi, P. Vasseur, and A. Bahloul, Int. Commun. Heat Mass Transfer 32, 770 (2005). https://doi.org/10.1016/j.icheatmasstransfer.2004.10.007

19. N. Ahmed and D. Kalita, Int. J. Math. Sci. Eng. Appl. 3, 267 (2009).

20. N. Ahmed and H. Kalita, Indian J. Sci. Technol. 3, 919 (2012).

21. M. Ali and M. S. Alam, J. Eng. Appl. Sci. 9, 361 (2014).

22. A. J. Chamkha and A. Ben-Nakhi, Heat and Mass Transfer 44, 845 (2008). https://doi.org/10.1007/s00231-007-0296-X

23. O. A. Bég, T. A. Bég, A. Y. Bakier, and V. R. Prasad, Int. J. Appl. Math. Mech. 5, 39 (2009).

24. C. Y. Cheng, Transp. Porous Media 94, 123 (2012). https://doi.org/10.1007/s11242-012-9992-1

25. B. K. Sharma, S. Gupta, V. Vamsikrishna, and R. J. Bhargavi, Afrika Mathematika 25, 799 (2014). https://doi.org/10.1007/s13370-013-0154-6

26. C. Kalyani, M. C. K. Reddy, and N. Kishan, Am. Chem. Sci. J. 7, 150 (2015). https://doi.org/10.9734/ACSJ/2015/16257

27. S. Hazarika and S. Ahmed, J. Sci. Res. 13, 31 (2021). https://doi.org/10.3329/jsr.v13i1.47458

28. D. Kalita, S. Hazarika, and S. Ahmed, JP J. Heat Mass Trans. 20, 105 (2020). https://doi.org/10.17654/HM020020105

29. B. Phukan, G. C. Hazarika, and S. Ahmed, J. Eng. Phys. Thermophysics 93(1), 178 (2020). https://doi.org/10.1007/s10891-020-02106-7

30. S. Ahmed, J. Zueco, and L. M. López-González, Int. J. Heat Mass Transfer 104, 409 (2017). https://doi.org/10.1016/j.ijheatmasstransfer.2016.07.076

31. K. Kalita and S. Ahmed, J. Eng. Phys. Thermophys. 90, 918 (2017). https://doi.org/10.1007/s10891-017-1639-1

32. S. Ahmed and K. Kalita, J. Naval Architect. Marine Eng. 11, 39 (2014). https://doi.org/10.3329/jname.v11i1.10269

33. S. Ahmed, G. C. Hazarika, and G. Gogoi, J. Naval Architect. Marine Eng. 17, 183 (2021). http://dx.doi.org/10.3329/jname.v17i2.48477

34. S. Hazarika, S. Ahmed, and S.-W. Yao, Appl. Nanosci. (2021) (In press). https://doi.org/10.1007/s13204-020-01655-w 